\title{
OPTIMUM DESIGN OF 4-UPU PARALLEL ROBOT USING CONDITION NUMBER
}

\author{
A. Chandrashekhar \\ Asst. Prof, Mechanical Engineering Department, \\ The ICFAI Foundation for Higher Education, Hyderabad, India \\ G. Satish Babu \\ Professor, Department of Mechanical Engineering, \\ JNTUH College of Engineering Hyderabad, India
}

\begin{abstract}
In this paper condition number is used as a measure to identify optimum structures in a parallel robot. Several structures have been analyzed in a 4 UPU robot in order to identify most optimum configurations. This method has led our research analysis to identify isotropic or nearly isotropic configurations. Matlab code is developed to obtain results which have been represented in a graphical form.
\end{abstract}

Key words: Performance Index, Isotropic Configurations, Condition Number

Cite this Article: A. Chandrashekhar and G. Satish Babu. Optimum Design of 4-Upu Parallel Robot Using Condition Number, International Journal of Industrial Engineering Research and Development, 6(2), 2015, pp. 34-38. https://iaeme.com/Home/issue/IJIERD?Volume $=6 \&$ Issue $=2$

\section{INTRODUCTION}

Salisbury and Craig (1) are the first to apply the condition number idea to systems. They have utilized the condition number of the Jacobian Matrix as an enhancement measure to get perfect measurements for the systems with the two revolute joints and Stanford JPL Articulated hand. Kazuhiro Kosuge and Katsuhisa Furuta (2) utilized the opposite of the condition number of Jacobian Matrix as a measure of kinematic controllability. Klein and Blaho (3) proposed a few smoothness measures for an enhancing stance for a given end-effector position and for planning the ideal connection lengths of an arm. The four measures Determinant, Condition number, Minimum solitary esteem and Joint extent accessibility are resolved for the whole reach of the planar three connection revolute jointed controller. Lenient M.Gosselin (4) proposed new ability records for planar and spatial controllers. These records depend on the condition number of the Jacobian Matrix of the controllers. He planned kinematic comparisons in a manner that prompts skill records that are edge invariant, 
where as existing lists are influenced by a scaling of the controller. He proposed two records for planar controllers.

Gosselin (5) proposed new skill files that can be connected to planar and spatial controllers. These lists depend on the condition number of the Jacobian framework of the controllers. As the current lists are influenced by a scaling of the controller, another detailing of these mathematical statements is proposed by them to stay away from the issue of dimensional reliance. Two ability files, taking into account a repetitive definition of the speed mathematical statements and least number of parameters are introduced by them.

In this paper 4 UPU robot has been studied in order to identify optimum configurations based on condition number.

\section{MEASURE OF ISOTROPIC CONFIGURATIONS}

\subsection{Condition Number}

$$
\mathrm{k}=\|J\|\left\|J^{-T}\right\|
$$

Points in the workspace that minimize the condition number of the Jacobian matrix are the best conditioned to minimize error propagation from input torques to output forces. The best conditioning possible occurs when condition number is equal to one. The best conditioned points in the workspace are called as isotropic points by J.K. Salisbury and J.J. Craig. The minimization of condition number in the manipulators workspace could serve as an optimization criterion in link design.

\section{4-UPU PARALLEL MANIPULATOR}

The mechanism under discussion consists of two platforms connected to each other by eight serial chains as shown in Fig 1 One of the platforms is fixed to the ground while the other one is free. Each of the eight serial chains has a total of six degrees of freedom. All the eight serial chains are connected to the top platform by passive spherical joints. Each chain is connected to the bottom platform by two active perpendicular revolute, forming the universal joint, also each chain has a passive prismatic joint $\mathrm{L}_{\mathrm{i}}$ connecting the passive ball joint at $\mathrm{P}_{\mathrm{i}}$ to the active universal joint at $\mathrm{O}_{\mathrm{i}}$ as shown in Fig. 1.

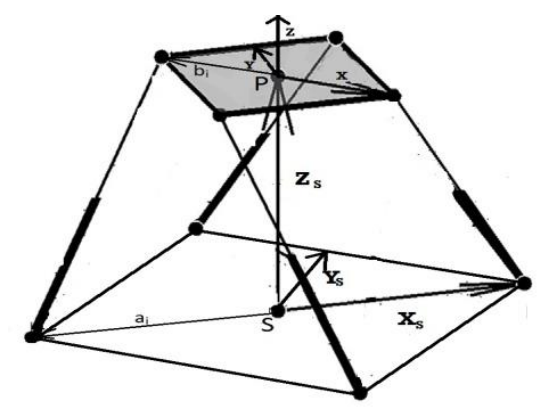

Figure 1 Kinematic Sketch of 4 UPU Parallel Robot

\subsection{Jacobian Analysis}

The instantaneous twist, $\$_{P}$, of the moving platform can be expressed as

$\$_{P}=\hat{\theta}_{1} \hat{\$}_{1, i}+\hat{\theta}_{2} \hat{\$}_{2, i}+\hat{d}_{3} \hat{\$}_{3, i}+\hat{\theta}_{4} \hat{\$}_{4, i}+\hat{\theta}_{5} \hat{\$}_{5, i}$ 
for $i=1,2, \ldots \ldots .5$

where

$\hat{\$}_{1, i}=\left(\begin{array}{l}s_{1, i} \\ \left(b_{i}-d_{i}\right) \times s_{1, i}\end{array}\right)$

$\hat{\$}_{2, i}=\left(\begin{array}{l}s_{2, i} \\ \left(b_{i}-d_{i}\right) \times s_{2, i}\end{array}\right)$

$\hat{\$}_{3, i}=\left(\begin{array}{l}0 \\ s_{3, i}\end{array}\right)$

$\hat{\$}_{4, i}=\left(\begin{array}{l}s_{4, i} \\ b_{i} \times s_{4, i}\end{array}\right)$

$\hat{\$}_{5, i}=\left(\begin{array}{l}s_{5, i} \\ b_{i} \times s_{5, i}\end{array}\right)$

Where $\mathrm{s}_{\mathrm{j}, \mathrm{i}}$ is a unit vector along the $\mathrm{j}^{\text {th }}$ joint axis of the $\mathrm{i}^{\text {th }} \operatorname{limb}, b_{i}=\overline{P B}_{i}$ and $d_{i}=\bar{A}_{i} B_{i}=d_{3, i} S_{3, i}$

The reciprocal screw, denoted as $\hat{\$}_{r, 1, i}$ is pointing in a direction perpendicular to the joint axes of the universal joint.

$$
\begin{aligned}
& \hat{\$}_{r, 1, i}=\left(\begin{array}{l}
0 \\
n_{i}
\end{array}\right) \\
& \hat{\$}_{r, 1, i}^{T}=0 \\
& J_{C} \$_{P}=0
\end{aligned}
$$

Where

$$
J_{c}=\left(\begin{array}{ll}
n_{1}{ }^{T} & 0_{1 \times 3} \\
n_{2}{ }^{T} & 0_{1 \times 3} \\
n_{3}{ }^{T} & 0_{1 \times 3} \\
n_{4}{ }^{T} & 0_{1 \times 3}
\end{array}\right)
$$

Reciprocal to all passive joint screws of the ith limb can be identified as zero pitch screw along the line passing through the two universal joints

$$
\hat{\$}_{r, 2, i}=\left(\begin{array}{l}
s_{3, i} \\
b_{i} \times s_{3 \times i}
\end{array}\right)
$$


$\hat{\$}_{r, 2, i}^{T} \$_{P}=d_{3, i}$

$J_{x} \$_{P}=q$

Where

$J_{x}=\left(\begin{array}{cc}\left(b_{1} \times s_{3,1}\right)^{T} & s_{3,1}^{T} \\ \left(b_{2} \times s_{3,2}\right)^{T} & s_{3,2}^{T} \\ \left(b_{3} \times s_{3,3}\right)^{T} & s_{3,3}^{T} \\ \left(b_{4} \times s_{3,4}\right)^{T} & s_{3,4}^{T}\end{array}\right)$

$q=\left[d_{3,1}, d_{3,2}, d_{3,3}, d_{3,4}\right]^{T}$

Therefore $q_{0}=J \$_{p}$

Where

$J=\left(\begin{array}{ll}\left(b_{1} \times s_{3,1}\right)^{T} & s_{3,1}^{T} \\ \vdots & \vdots \\ \left(b_{4} \times s_{3,4}\right)^{T} & s_{3,4}^{T} \\ n_{1}^{T} & 0_{1 \times 3} \\ \vdots & \vdots \\ n_{4}^{T} & 0_{1 \times 3}\end{array}\right)$

\section{RESULTS}

Using Matlab the following results have been obtained for 4 UPU spatial manipulators. The results regarding optimum structures in which nearly isotropic configurations were identified have been presented in this section.

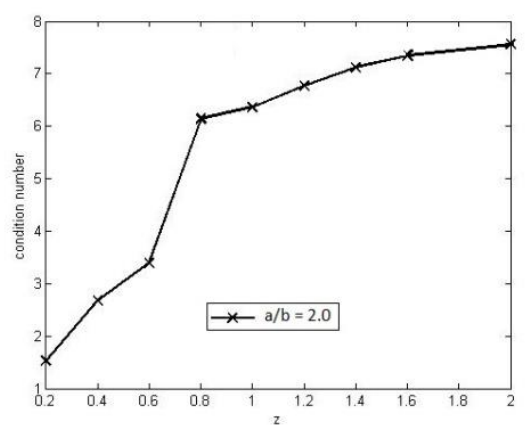

Figure 2 Graph illustrating condition number vs Reach $(\mathrm{a} / \mathrm{b}=2.0)$

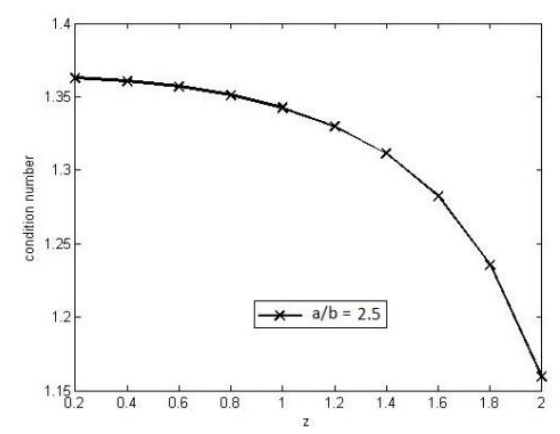

Figure 3 Graph illustrating condition number vs Reach $(\mathrm{a} / \mathrm{b}=2.5)$ 


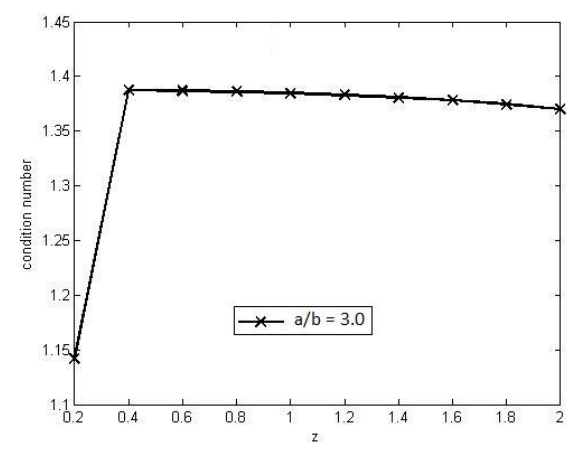

Figure 4 Graph illustrating condition number vs Reach $(\mathrm{a} / \mathrm{b}=3.0)$

\section{CONCLU SION}

Using condition number as a performance index, several structures have been analyzed in order to identify the nearly isotropic configurations. Finally in three structures nearly isotropic configurations have been found.

\section{REFERENCES}

[1] J. Kenneth Salisbury and John J. Craig. "Articulated Hands: Force Control and Kinematic Issues," The international Journal of Robotics Research, Vol.1, No. 1, pp 4-17, spring 1982. doi:10.1177/027836498200100102

[2] K. Kosuge and K. Furuta, "Kinematic and dynamic analysis of robot arm," Proceedings. 1985 IEEE International Conference on Robotics and Automation, St. Louis, MO, USA, 1985, pp. 1039-1044, doi: 10.1109/ROBOT.1985.1087278.

[3] Klein and Blaho. "Dexterity Measures for the Design and Control of Kinematically Redundant Manipulators," The international Journal of Robotics Research, Vol. 6, No. 2, pp72-83, summer 1987.

[4] Clement M. Gosselin. "Dexterity Indices for Planar and Spatial Robotic Manipulators," Proceedings., IEEE International Conference on Robotics and $A u$ tomation, Cincinnati, OH, USA, 1990, pp. 650-655 vol.1, doi: 10.1109/ROBOT. 1990.126057.

[5] Clement M. Gosselin. "The optimum design of robotic manipulators using dexterity indices," Robotics and Autonomous Systems, 9(4), pp 213-226 (1992). https://doi.org/10.1016/0921-8890(92)90039-2

[6] Chandrashekhar and G. Satish Babu, Force Isotropy of Three-Limb Spatial Parallel Manipulator. International Journal of Mechanical and Engineering Technology 6(6), 2015, pp. 01-08.

[7] A. Harish and G. Satish Babu, Manipulability Index of A Parallel Robot Manipulator, International Journal of Mechanical and Engineering Technology 6(6), 2015, pp. 9 - 17. 